Computer Optics and Nanophotonics

\title{
20 YEARS WITHOUT IOSIF NORAIROVICH SISSAKIAN
}

\author{
V.A. Danilov, N.I. Petrov \\ Scientific and Technological Centre of Unique Instrumentation, the Russian Academy of Sci- \\ ences, Moscow, Russian
}

\begin{abstract}
The report provides an overview of key scientific results of Professor I. N. Sissakian (08.03.1938 - 09.11.1995), and describes the development of the scientific direction "computer optics" after his untimely death.
\end{abstract}

Keywords: computer optics, diffractive optics, focusator, modan

Citation: Danilov VA, Petrov NI. 20 years without Iosif Norairovich Sissakian. CEUR Workshop Proceedings, 2016; 1638: 236-248. DOI: 10.18287/16130073-2016-1638-236-248

\section{Introduction}

It's been 20 years since the day of the untimely death of the chief designer Professor Iosif Sissakian of the Central Design Bureau of Unique Instrumentation of the Russian Academy of Sciences (CDB UI RAS) (currently - Scientific technological center of Unique Instrumentation of the Russian Academy of Sciences - STC UI RAS) (March 8, 1938 - November 9, 1995) [1-2]. The report discusses the scientific results of I.N. Sissakian (Fig. 1) in the area of optics and the development of his ideas in our days.

\section{Computer optics}

In post-graduate school I.N. Sissakian, along with Eugene L. Feinberg and D.S. Chernavskii, has published a number of theoretical works, based on the development of the hydrodynamical theory of Landau. After defending his thesis, I. N. Sissakian had changed the subject, focusing on optics. Cooperation with the group of Professor V.A. Soifer [3] from the Kuibyshev aviation Institute has led to the emergence of a new scientific field, called "computer optics" [4]. Elements of computer optics were calculated on the basis of solving the inverse problem of diffraction and manufactured by the methods of microelectronics or on CNC machines. I. N. Sissakian organized regular workshops on computer optics. Upon the results of the first (Zvenigorod, 1986) workshop the international collection "Computer Optics" [5] edited by A.M. Prokho- 
rov and E.P. Velikhov is started out. Now this is a journal, editor in chief is V.A. Soifer [6].

The first elements of computer optics were the elements for arbitrary transformation of the form of the wave front [7-8]. The Novosibirsk scientists from the Institute of automation and electrometry of SB RAS continue these studies in the laboratory headed by Professor A.G. Poleshchuk. They created the equipment to control the aspherical surface of a multi-meter telescope [9-10]. In 1988 on the initiative of I. N. Sissakian (decision of the Presidium of the USSR Academy of Sciences) the Kuibyshev branch of CDB UI of the USSR Academy of Sciences was established headed by V. A. Soifer (since 1993 - Image Processing Systems Institute of RAS - IPSI RAS [11-12]). In 1992 a team consisting of I. N. Sissakian, V. A. Soifer, V. P. Shorin, V. A. Barvinok, V. I. Bogdanovich, V. I. Mordasov, A. G. Tsidulko was awarded the State prize of Russia for outstanding achievements in science and technology ("for development of laser technologies and their implementation to create new aviation and space technology").

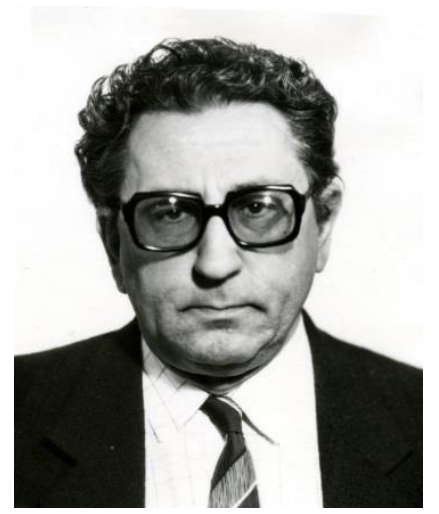

Fig. 1. Professor Iosif Norairovich Sissakian (March 8, 1938 - November 9, 1995)

Under the direction of I. N. Sissakian several PhD and doctoral dissertations were protected in the field of computer optics. In addition to Moscow and Samara, computer optics is developing in Novosibirsk, St. Petersburg, Chernogolovka, Kazan and Penza. A number of his students are now successfully working abroad. I. N. Sissakian supported work of Penza group under the direction of G. I. Greisukh as for the gradient-index, and the diffractive imaging optics [13-14].

\section{Focusators of laser radiation}

The first focusators were axisymmetric elements (diffraction axicons), creating coaxis line and the circle [15-16]. Contact of I. N. Sissakian with physicians has led to the formulation of focusing of laser radiation into the cross for the operation of radial keratotomy [17]. Then the task was the synthesis of focusators in an arbitrary curve in the focal plane with a given intensity distribution on the curve. Focusators were created from the visible to millimeter range [18-24]. The problem of focusing in a flat region and a sloped line segments was considered [25-31]. Focusators were investi- 
gated theoretically and experimentally [32-41]. New methods for the diffractive microrelief formation were developed to create the focusators [42-51].

In 1989 focusator in a transverse cut with a high intensity distribution at the edges was successfully tested in the US in the Institute "General Motors" for heat strengthening of steel using $3 \mathrm{~kW}$ laser of the company "Spectro-physics". Laser technologies with the use of focusators are actively developing [52-53]. The calculation methods were used for focusing surface electromagnetic waves [54-58].

\section{Formation of light beams with remarkable properties}

Modans which spatially divide transverse modes of laser beams were created [59-62]. Such optical elements are effective for the analysis of a modal content of radiation propagating in different media as well as for creation of high-sensitive fiber-optics sensors. Thus, in [60], the modans consistent with the Gauss-Laguerre modes were used to determine the modal coefficients in a graded-index waveguide. In [61], the modans with transmission proportional to the superposition of orthogonal FourierBessel functions were proposed to determine the characteristics of aerosol medium. It was shown that the coefficients of the decomposition of scattered light by aerosol particles into the series of Fourier-Bessel functions define the relative concentration of particles or the distribution function of particle sizes. The coefficients of expansion into the series of Bessel functions of different orders define the moments of the distribution function. Moreover, the use of spatial filters, carrying out these transformations, allow the parameters of particles to be directly determined. Investigations in this area are ongoing both with the aim of increasing the sensitivity of fiber-optic sensors [63] and for increasing of transmission capacity and protection of data in optical communication lines [64].

The paper [65] was among the first works in the area of singular optics devoted to the elements forming the beams describing by the Bessel functions. Then a number of elements with unique properties was proposed [66-68]. Optical antennas create the radiation in the form of petals keeping their form and propagation angle at long distances. This made it possible to create lighting devices for various applications [6974].

\section{Propagation of light in inhomogeneous media}

The methods of quantum theory (quantum field theory, quantum statistics, quantum mechanics) were proposed by I.N. Sisakian to solve the tasks of propagation of radiation in inhomogeneous media. In particularly, the coherent states and integrals of motion methods were used for description of propagation of light beams in longitudinally inhomogeneous graded-index waveguides [75-76]. This allowed us to investigate the physics of wave processes both in optical waveguides and natural waveguide channels [77-79]. The quantum-mechanical methods of coherent states allowing the calculation of the average values with the help of operator approach were used to investigate the evolution of the parameters of light beams. The whole dynamics of the system is transferred to the operators in this approach. This allows us to investigate 
the evolution of the characteristics of the propagating beam with the help of pure algebraic procedure, that is without using explicit expressions for field wavefunctions and without the calculation of any integrals. Coherent states correspond to the wave beams localized in the neighbourhood of the geometrical ray. Such states allow us to introduce in natural way the term of the width of geometrical ray and clearly trace the connection between the wave and geometrical descriptions. The concept of choice of quantum-mechanical formalism in the theory of waveguides is the following. As was shown in [75], the Maxwell equations for the scalar wave paraxial beams may be reduced with high accuracy to a parabolic-type equation. This approximation allows us to apply well developed quantum-mechanical methods to study wave propagation in inhomogeneous media, since the parabolic equation formally is quite similar to the Schrodinger equation in quantum mechanics for a particle moving in a potential well with the parameters depending on time. Only some corresponding redefinitions of parameters need to be carried out. The time plays the role of the longitudinal coordinate and, instead of the Planck constant we have the free-space wavelength. The potential is determined by the refractive index of the medium. The close connection between wave mechanics of particles and optics of light beams has been discussed in detail by many workers. The efficiency of the application of the coherent states representation and density matrix in the tasks of propagation of partially-coherent radiation in inhomogeneous media was shown by I.N. Sisakian and his followers [80-82]. The behaviour of spatial coherence of optical fields in the media with general-squareindex and arbitrary regular longitudinal inhomogeneity was investigated. The analytical expressions for the parameters describing the spatial coherency of fields in such media, in particular, for the correlation radius and the width of partially-coherent beam were obtained. It was shown, that the coherent properties of light can be controlled with the help of variation of the laws of longitudinal inhomogeneity. Quantumtheoretical methods were applied also for investigation of the effects of nonparaxiality [83-87] and depolarization [88-90] of radiation in graded-index media. In [85] the nonparaxial focusing of wave beams in a graded-index medium was studied. The minimum dimensions limited by the nonparaxial effects of the wave-beam focusing area were determined. These effects lead to a significant change in the transverse distribution of the light intensity in the focusing area and to an asymmetric distribution of the field intensity in the longitudinal direction. In [87] the remote focusing of light in a graded-index medium via mode interference is demonstrated using exact analytical solutions of the wave equation. Strong focusing of a light beam occurs at extremely long distances and this repeats periodically with distance. High efficiency transfer of a strongly focused subwavelength spot through optical waveguide over large distances takes place with a period of revival. Results obtained may be of great importance in biology and medicine, optical recording and microscopy, and could be exploited in various applications such as novel endoscopes, sensors and imaging systems. In [88-90] the polarization effects at the propagation of light in a multimode graded-index optical fiber are considered using the method of coherent states. In [9194] these methods were used for consideration of the effects of spin-orbit interaction of light in optical waveguides with a cylindrical symmetry. Propagation of polarized vortex light beams in a rotationally symmetric graded-index optical waveguide is investigated by solving analytically the three-component field Maxwell's equations. An operator approach to calculate the average values describing the propagating 
beams is developed. In [94] the influence of polarization (spin) and orbital angular momentum on the characteristics of spiral (vortex) light beams at the propagation in a graded-index fiber is studied.

\section{Automation of scientific research}

I.N. Sissakian paid great attention to the development of information technologies and automation of scientific research, as well as to optical instrumentation. The second edition of the international proceedings "Computer optics" prepared by him for printing was entirely devoted to the problems of automation of scientific research [95]. He raised and organized the solution of tasks of computing [96-97] and optical [98103] experiments, paid significant attention to the development of asymptotic methods in computer optics [104-105]. In all of these areas the studies are being carried out to the present time [106-111]. Works in the field of optical instrumentation are continuing in the STC UI RAS and IPSI RAS. For example, the development of hyperspectrometers: on the basis of acousto-optics - in STC UI RAS [112], and on the basis of spectral filters and diffractive optical elements - in IPSI RAS [113-116].

\section{Conclusion}

Practical implementation of elements of computer optics in modern digital cameras demanded the suppression of spectral and angular dependences of their diffraction efficiency. Various aspects of this task were presented, in particular, in the works [117-119]. The results obtained allowed us to define the conditions under which the high image quality (and, in particular, the lack of a halo) can be achieved using the elements in an optical system with single-layer or double-layer relief-phase microstructure.

Methods of computer optics are used for creation of new elements for the control of light [120], and technologies of manufacturing of holographic gratings [121]. In [120] a high-efficiency subwavelength diffractive beam combiner operating in a visible spectral range is designed, fabricated, and demonstrated. Such a device combines red, green, and blue color beams into one output light beam. Diffraction efficiencies of different types of gratings are calculated for various materials, incidence angles, and polarizations of light. It is shown that the plasmon resonance via a grating coupling occurs at the determined conditions. Subwavelength gratings with a period of $400 \mathrm{~nm}$ are fabricated and tested using laser and laser diode sources. This type of color combiner can be useful in many application areas, such as picoprojectors, where efficiency and compact size are crucial. It was shown that such gratings have a high efficiency and can significantly reduce the size of the projection devices used in mobile phones, etc.

Modern computational and technological capabilities allow colleagues and students of I. N. Sissakian to transfer from the tasks of diffractive optics to the problems of diffractive nanophotonics [122-131]. 
These results open up the prospects of solving one of the main tasks of computer optics - implementation of optical calculations [124-127] and lay the intellectual foundations of advanced information technologies [131].

Finally, we mention two practical applications of computer optics elements: protective holograms [132-133] and optical filters for professional and amateur photography [134].

\section{References}

1. THE JUMP BY ROLLING. The memory of Professor I. N. Sissakian / ed. by Soifer VA, Khomich VYu. Dubna: JINR; 2005. [in Russian]

2. Kazanskiy NL. Professor Iosiph Norairovich Sisakyan -70 years old. Vestnik of the Samara State Aerospace University, 2008; 2(15): 9-34. [in Russian]

3. Sokolov VO. On the 70th Birthday of corresponding member of the Russian Academy of Sciences Victor A. Soifer. CEUR Workshop Proceedings, 2015; 1490: 1-8. DOI: 10.18287/1613-0073-2015-1490-1-8.

4. Sisakyan IN, Soifer VA. Computer optics. Achievements and problems. Computer Optics, 1989; 1(1): 3-12.

5. Kolomiets EI. Analysis of activity of the scientific journal Computer Optics. CEUR Workshop Proceedings, 2015; 1490: 138-150. DOI: 10.18287/1613-0073-2015-1490-138-150.

6. Soifer VA. Quo vadis. Computer Optics, 2014; 38(4): 589.

7. Golub MA, Zhivopistsev ES, Karpeev SV, Prokhorov AM, Sissakian IN, Soifer VA. Generation of aspherical wavefronts using computer generated holograms. Doklady AN SSSR, 1980; 253(5): 1104-1108.

8. Golub MA, Kazanskiy NL, Sisakyan IN, Soifer VA. Wavefronts forming by computergenerated optical elements. Proceedings of SPIE - The International Society for Optical Engineering, 1990; 1183: 727-750. DOI: 10.1117/12.963891.

9. Poleshchuk AG, Nasyrov RK. Aspherical wavefront shaping with combined computer generated holograms. Optical Engineering, 2013; 52(9): 091709.

10. Poleshchuk AG, Sedukhin AG. Diffraction technique for testing the resolution and sensitivity of Hartmann and Shack-Hartmann sensors. Optics Letters, 2015; 40(21): 5050-5053.

11. Kolomiets EI. Analysis of the scientific and organizational results of the Image Processing Systems Institute of the RAS. CEUR Workshop Proceedings, 2015; 1490: 309-326. DOI: 10.18287/1613-0073-2015-1490-309-326.

12. Kazanskiy NL. Research \& education center of diffractive optics. Proceedings of SPIE, 2012; 8410: 84100R. DOI: 10.1117/12.923233.

13. Greisukh GI, Bobrov ST, Stepanov SA. Optics of diffractive and gradient-index elements and systems. Bellingham: SPIE Press, 1997.

14. Greisukh GI, Ezhov EG, Sidyakina ZA, Stepanov SA. Design of plastic diffractiverefractive compact zoom lenses for visible-near-IR spectrum. Applied Optics, 2013; 52(23): 5843-5850

15. Golub MA, Karpeev SV, Prokhorov AM, Sissakian IN, Soifer VA. Focusing of coherent radiation into a given region of space using computer synthesized holograms. Technical Physics Letters, 1981; 7(10): 618-623.

16. Golub MA, Degtyareva VP, Klimov AN, Popov VV, Prokhorov AM, Sissakian IN, Soifer VA. Computer-aided synthesis of focusing elements for $\mathrm{CO}_{2}$-laser. Technical Physics Letters, 1982; 8(8): 449-451. 
17. Akopyan VS, Danileiko YuK, Danilov VA, Naumidi LP, Popov VV, Sissakian IN. Use of planar axially asymmetric focusers in laser ophthalmic surgery. Soviet Journal of Quantum Electronics, 1985; 15(2): 262-263.

18. Danilov VA, Popov VV, Prokhorov AM, Sagatelyan DM, Sissakian IN, Soifer VA. Synthesis of optical elements creating a focal line of arbitrary shape. Technical Physics Letters, 1982; 8(13) 810-815.

19. Danilov VA, Popov VV, Prokhorov AM, Sagatelyan DM, Sissakian EV, Sissakian IN, Soifer VA. Optical elements focusing coherent radiation into arbitrary focal line. Preprint FIAN SSSR No. 69. - Moscow; 1983. [in Russian]

20. Goncharsky AV, Danilov VA, Popov VV, Prokhorov AM, Sissakian IN, Soifer VA, Stepanov VV. Solution of the inverse problem of focusing of laser radiation into an arbitrary curve. Doklady AN SSSR, 1983; 273(3): 605-608.

21. Goncharsky AV, Danilov VA, Popov VV, Prokhorov AM, Sissakian IN, Soifer VA, Stepanov VV. Devices for focusing laser radiation incident at an angle. Soviet Journal of Quantum Electronics, 1984; 14(1): 108-109.

22. Goncharsky AV, Danilov VA, Popov VV, Prokhorov AM, Sissakian IN, Soifer VA, Stepanov VV. Planar focusing components for the visible range. Soviet Journal of Quantum Electronics, 1986; 16(3): 432-433.

23. Bulatov ED, Vinogradov EA, Gridin SA, Golovanov VI, DanilenkoAA, Danilov VA, Irisova NA, Prokhorov AM, Sissakian IN. Focusing systems for the millimeter range. Soviet Journal of Quantum Electronics, 1989; 19(1): 113-115.

24. Golub MA, Sisakian IN, Soifer VA. Infra-red radiation focusators. Optics and Lasers in Engineering, 1991; 15(5): 297-309.

25. Goncharsky AV, Danilov VA, Popov VV, Prokhorov AM, Sissakian IN, Stepanov VV. On one inverse problem of synthesis of optical elements. Doklady AN SSSR, 1986; 291(3) 591-595.

26. Danilov VA, Kulkin KA, Sissakian IN. Focusators into segment constituting arbitrary angle with the optical axis. Computer Optics, 1992; 10-11: 48-68. [in Russian]

27. Doskolovich LL, Kazanskiy NL, Sissakian IN, Soifer VA, Kharitonov SI. Focusing of laser radiation on three-dimensional surfaces of rotation. Computer Optics, 1992; 12: 8-14. [in Russian]

28. Danilov VA, Kulkin KA, Sissakian IN. Focusator into figures composed of spatial curves. Computer Optics, 1993; 13: 3-11. [in Russian]

29. Danilov VA, Kinber BE, Shishlov AV. Theory of coherent focusers. Computer Optics, 1989; 1(1): 29-37.

30. Danilov VA, Dubov MV. Effects of distortions in incident-beam intensity of focuser operation. Computer Optics, 1989; 1(1): 39-49.

31. Kazanskiy NL, Kotlyar VV, Soifer VA. Computer-aided design of diffractive optical elements. Optical Engineering, 1994; 33(10): 3156-3166.

32. Kazanskiy NL, Soifer VA. Diffraction investigation of geometric-optical focusators into segment. Optik - International Journal for Light and Electron Optics, 1994; 96(4): 158162.

33. Doskolovich LL, Kazanskiy NL, Soifer VA, Tzaregorodtzev AYe. Analysis of quasiperiodic and geometric optical solutions of the problem of focusing into an axial segment. Optik - International Journal for Light and Electron Optics, 1995; 101(2): 37-41.

34. Doskolovich LL, Golub MA, Kazanskiy NL, Khramov AG, Pavelyev VS, Seraphimovich PG, Soifer VA, Volotovskiy SG. Software on diffractive optics and computer generated holograms. Proceedings of SPIE, 1995; 2363: 278-284. DOI: 10.1117/12.199645. 
35. Doskolovich LL, Kazansky NL, Kharitonov SI, Soifer VA. A method of designing diffractive optical elements focusing into plane areas. Journal of Modern Optics, 1996; 43(7): 1423-1433. DOI: 10.1080/09500349608232815.

36. Kazanskiy NL, Kharitonov SI, Soifer VA. Application of a pseudogeometrical optical approach for calculation of the field formed by a focusators. Optics \& Laser Technology, 1996; 28(4): 297-300. DOI: 10.1016/0030-3992(95)00103-4.

37. Doskolovich LL, Kazanskiy NL, Soifer VA, Perlo P, Repetto P. Design of DOEs for wavelength division and focusing. Journal of Modern Optics, 2005; 52(6): 917-926. DOI: 10.1080/09500340512331313953.

38. Kazanskiy NL. Asymptotic research in computer optics. CEUR Workshop Pro-ceedings, 2015; 1490: 151-161. DOI: 10.18287/1613-0073-2015-1490-151-161.

39. Goncharsky AV, Danilov VA, Popov VV, Prokhorov AM, Sissakian IN, Soifer VA, Stepanov VV. Focusing elements for laser material processing. Technical Physics Letters, 1985; 11(23): 1428-1432.

40. Ageshin SF, Azarov AA, Popov VV, Sisakyan IN. Use of focusators in laser assisted material processing. Computer Optics, 1990; 2(1): 81-83.

41. Doskolovich LL, Kazanskiy NL, Kharitonov SI, Usplenjev GV. Focusators for laserbranding. Optics and Lasers in Engineering, 1991; 15(5): 311-322. DOI: 10.1016/01438166(91)90018-O.

42. Babin SV, Danilov VA. Variable shaped electron-beam lithography application to subwavelength and computer generated diffractive optics fabrication. Journal of Vacuum Science \& Technology B, 1995; B13(6): 2767-2771.

43. Volkov AV, Kazanskiy NL, Moiseev OYu, Soifer VA. A Method for the Diffractive Microrelief Formation Using the Layered Photoresist Growth. Optics and Lasers in Engineering, 1998; 29(4-5): 281-288.

44. Babin SV, Danilov VA. Data preparation and Fabrication of DOE using Electron-beam Lithography. Optics and Lasers in Engineering, 1998; 29(4-5): 307-324.

45. Kazanskiy NL, Uspleniev GV, Volkov AV. Fabricating and testing diffractive optical elements focusing into a ring and into a twin-spot. Proceedings of SPIE, 2000; 4316: 193199. DOI: $10.1117 / 12.407678$.

46. Kazanskii NL, Kolpakov VA, Kolpakov AI. Anisotropic Etching of $\mathrm{SiO}_{2}$ in High-Voltage Gas-Discharge Plasmas. Russian Microelectronics, 2004; 3(3): 169-182. DOI: 10.1023/B:RUMI.0000026175.29416.eb.

47. Pavelyev VS, Borodin SA, Kazanskiy NL, Kostyuk GF, Volkov AV. Formation of diffractive microrelief on diamond film surface. Optics \& Laser Technology, 2007; 39(6): 12341238. DOI: 10.1016/j.optlastec.2006.08.004.

48. Bezus EA, Doskolovich LL, Kazanskiy NL. Interference pattern formation in evanescent electromagnetic waves using waveguide diffraction gratings. Quantum Electronics, 2011; 41(8): 759-764. DOI: 10.1070/QE2011v041n08ABEH014500.

49. Abul'khanov SR, Kazanskii NL, Doskolovich LL, Kazakova OY. Manufacture of diffractive optical elements by cutting on numerically controlled machine tools. Russian Engineering Research, 2011; 31(12): 1268-1272. DOI: 10.3103/S1068798X11120033.

50. Kazanskiy NL, Kolpakov VA, Podlipnov VV. Gas discharge devices generating the directed fluxes of off-electrode plasma. Vacuum, 2014; 101: 291-297. DOI: 10.1016/j.vacuum.2013.09.014.

51. Kazanskiy NL, Moiseev OYu, Poletayev SD. Microprofile Formation by Thermal Oxidation of Molybdenum Films. Technical Physics Letters, 2016; 42(2): 164-166. DOI: $10.1134 /$ S1063785016020085. 
52. Kazanskiy NL, Murzin SP, Tregub VI. The optical system for the selective laser sublimation of the components of the metal alloys. Computer Optics, 2010; 34(4): 481-486.

53. Kazanskiy NL, Murzin SP, Osetrov EL., Tregub VI. Synthesis of nanoporous structures in metallic materials under laser action. Optics and Lasers in Engineering, 2011; 49(11): 1264-1267. DOI: 10.1016/j.optlaseng.2011.07.001.

54. Bezus EA, Doskolovich LL, Kazanskiy NL, Soifer VA, Kharitonov SI, Pizzi M, Perlo P. The design of diffractive optical elements to focus surface plasmons. Computer Optics, 2009; 33(2): 185-192.

55. Bezus EA, Doskolovich LL, Kazanskiy NL, Soifer VA, Kharitonov SI. Design of diffractive lenses for focusing surface plasmons. Journal of Optics, 2010; 12(1): 015001. DOI: 10.1088/2040-8978/12/1/015001.

56. Bezus EA, Doskolovich LL, Kazanskiy NL. Scattering suppression in plasmonic optics using a simple two-layer dielectric structure. Applied Physics Letters, 2011; 98(22): 221108. DOI: $10.1063 / 1.3597620$.

57. Bezus EA, Doskolovich LL, Kazanskiy NL, Soifer VA. Scattering in elements of plasmon optics suppressed by two-layer dielectric structures. Technical Physics Letters, 2011; 37(12): 1191-1095. DOI: 10.1134/S1063785011120030.

58. Bezus EA, Doskolovich LL, Kazanskiy NL. Low-scattering surface plasmon refraction with isotropic materials. Optics Express, 2014; 22(11): 13547-13554. DOI: 10.1364/OE.22.013547.

59. Golub MA, Prokhorov AM, Sissakian IN, Soifer VA. Synthesis of spatial filters for investigation of the transverse mode composition of coherent radiation. Soviet Journal of Quantum Electronics, 1982; 12(9): 1208-1209.

60. Garitchev VP, Golub MA, Karpeev SV, Krivoshlykov SG, Petrov NI, Sissakian IN, Soifer VA, Haubenreisser W, Jahn JU, Willsch R. Experimental investigation of mode coupling in a multimode graded-index fiber caused by periodic microbends using computergenerated spatial filters. Optics Communications, 1985; 55(6): 403-405.

61. Petrov NI, Sisakyan IN, Sisoyev VS. Computer-synthesized optical elements in diagnosis of aerosol systems. Computer Optics, 1990; 2(1): 89-90.

62. Sissakian IN, Soifer VA. Modans - optical elements for analysis and synthesis of laser mode structure. Computer Optics, 1990; 2(2): 109-113.

63. Karpeev SV, Pavelyev VS, Khonina SN, Kazanskiy NL, Gavrilov AV, Eropolov VA. Fibre sensors based on transverse mode selection. Journal of Modern Optics, 2007; 54(6): 833-844. DOI: 10.1080/09500340601066125.

64. Lyubopytov VS, Tlyavlin AZ, Sultanov AH, Bagmanov VH, Karpeev SV, Khonina SN, Kazanskiy NL. Mathematical model of completely optical system for detection of mode propagation parameters in an optical fiber with few-mode operation for adaptive compensation of mode coupling. Computer Optics, 2013; 37(3): 352-359.

65. Bereznyi AE, Prokhorov AM, Sissakian IN, Soifer VA. Bessel optics. Doklady Akad. Nauk SSSR, 1984; 274(3) 623-627.

66. Kotlyar VV, Kovalev AA, Soifer VA. Nondiffractive asymmetric elegant Bessel beams with fractional orbital angular momentum. Computer Optics, 2014; 38(1): 4-10.

67. Kotlyar VV, Kovalev AA, Skidanov RV, Soifer VA. Rotating elegant Bessel-Gauss beams. Computer Optics, 2014; 38(2): 162-170.

68. Soifer VA, Kotlyar VV, Doskolovich LL. Diffractive optical elements in nanophotonics devices. Computer Optics, 2009; 33(4): 352-368.

69. Golub MA, Kazanskiy NL, Prokhorov AM, Sissakian IN, Soifer VA. Synthesis of optical antennae. Computer Optics, 1989; 1(1): 25-28. 
70. Doskolovich LL, Kazanskiy NL, Soifer VA, Kharitonov SI, Perlo P. A DOE to form a line-shaped directivity diagram. Journal of Modern Optics, 2004; 51(13): 1999-2005. DOI: $10.1080 / 09500340408232507$.

71. Doskolovich LL, Kazanskiy NL, Bernard S. Designing a mirror to form a line-shaped directivity diagram. Journal of Modern Optics, 2007; 54(4): 589-597. DOI: 10.1080/0950034060102186.

72. Aslanov ER, Doskolovich LL, Moiseev MA, Bezus EA, Kazanskiy NL. Design of an optical element forming an axial line segment for efficient LED lighting systems. Optics Express, 2013; 21(23): 28651-28656. DOI: 10.1364/OE.21.028651.

73. Dmitriev AYu, Doskolovich DL, Doskolovich LL, Kazanskiy NL. Analytical calculation of the refractive optical elements for forming one-parametric radiation diagrams. Computer Optics, 2014; 38(2): 207-212.

74. Doskolovich LL, Borisova KV, Moiseev MA, Kazanskiy NL. Design of mirrors for generating prescribed continuous illuminance distributions on the basis of the supporting quadric method. Applied Optics, 2016; 55(4): 687-695. DOI: 10.1080/0950034060102186.

75. Krivoshlykov SG, Sissakian IN. Coherent states and light propagation in inhomogeneous media. Soviet Journal of Quantum Electronics, 1980; 10(3): 312-318.

76. Krivoshlykov SG, Petrov NI, Sissakian IN. Mode energy transformation between two connected multimode general square-law-index optical waveguides. Optical and Quantum Electronics, 1983; 15(3): 193-207.

77. Petrov NI, Sisakyan IN, Shvartsburg AB. Nonlinear-wave phenomena in the channel of lightning. Computer Optics, 1989; 6: 54-62. [in Russian]

78. Petrov NI, Sissakian IN. The emission of the lightning discharge. Computer Optics, 1993; 13: 65-75. [in Russian]

79. Petrov NI, Sissaian IN. Propagation of localized wave beams in the waveguide Earthionosphere. Radiotekhnika i Elektronika, 1994; 39(10): 1576-1586. [in Russian]

80. Krivoshlykov SG, Petrov NI, Sissakian IN. Spatial coherence of optical fields in longitudinally inhomogeneous media with quadratic refractive index profiles. Soviet Journal of Quantum Electronics, 1985; 15(3): 330-338.

81. Krivoshlykov SG, Petrov NI, Sissakian IN. Excitation of modes of waveguides with a quadratic refractive index profile by partially coherent radiation sources. Technical Physics, 1985; 55(9): 1763-1772.

82. Krivoshlykov SG, Petrov NI, Sissakian IN. Density-matrix formalism for partially coherent optical fields propagating in slightly inhomogeneous media. Optical and Quantum Electronics, 1986; 18: 253-264.

83. Krivoshlykov SG, Petrov NI, Sissakian IN. Correlated coherent states and propagation of arbitrary Gaussian beams in longitudinally homogeneous quadratic media exhibiting absorption or amplification. Soviet Journal of Quantum Electronics, 1986; 16(7): 933-941.

84. Krivoshlykov SG, Sauter EG. Propagation and focusing of nonparaxial Gaussian beams with spherical wave fronts in graded-index waveguides with polynomial profiles. JOSA A, $1993 ; 10(2): 262-268$.

85. Petrov NI. Focusing of beams into subwavelength area in an inhomogeneous medium. Optics Express, 2001; 9(12): 658-673.

86. Petrov NI. Macroscopic quantum effects for classical light. Physical Review A, 2014; 90(4): 043814.

87. Petrov NI. Remote focusing of a light beam. Laser Physics Letters, 2016; 13(1): 015101.

88. Petrov NI. Evolution of polarization in an inhomogeneous isotropic medium. Journal of Experimental and Theoretical Physics, 1997; 85(6): 1085-1093. 
89. Petrov NI. Evolution of Berry's phase in a graded-index medium. Physics Letters A, 1997; 234(4): 239-250.

90. Petrov NI. Depolarization of light in a graded-index isotropic medium. Journal of Modern Optics, 1996; 43(11):2239-2249.

91. Petrov NI. Beam shift in a graded-index optical fiber. Journal of Optics, 2013; 15(1): 014011.

92. Petrov NI. Splitting of levels in a cylindrical dielectric waveguide. Optics Letters, 2013; 38(12): $2020-2022$.

93. Petrov NI. Spin-orbit and tensor interactions of light in inhomogeneous isotropic media. Physical Review A, 2013; 88(2): 023815.

94. Petrov NI. Spin-dependent transverse force on a vortex light beam in an inhomogeneous medium. Journal of Experimental and Theoretical Physics Letters, 2016; 103(7): 504-509.

95. Bulatov ED, Otlivanchik EA, Otlivanchik MA, Sissakian IN, Suvorov AV. Personal computers in automation of scientific research. Computer Optics, 1987; 2: 35-41. [in Russian]

96. Golub MA, Kazanskii NL, Sisakyan IN, Soifer VA. Computational experiment with plane optical elements. Optoelectronics, Instrumentation and Data Processing, 1988; 24(1): 7889.

97. Golub MA, Doskolovich LL, Kazanskiy NL, Sissakian IN, Soifer VA, Kharitonov SI. Computational experiment with the focusator of Gaussian beam into a rectangle with a constant intensity. Computer Optics, 1990; 7: 42-49. [in Russian]

98. Aref'ev EY, Gilev AV, Golub MA, Kazanskiy NL, Karpeev SV, Sissakian IN, Soifer VA, Solovyev VS, Tikhonov DN, Uvarov GV. Experimental study of a flat optical element focusing into a ring. Computer Optics, 1989; 5: 49-54. [in Russian]

99. Doskolovich LL, Kazanskiy NL, Khonina SN, Skidanov RV, Heikkilä N, Siitonen S, Turunen J. Design and investigation of color separation diffraction gratings. Applied Optics, 2007; 46(15): 2825-2830. DOI: 10.1364/AO.46.002825.

100. Kazanskiy NL, Popov SB. Machine Vision System for Singularity Detection in Monitoring the Long Process. Optical Memory and Neural Networks (Information Optics), 2010; 19(1): 23-30. DOI: $10.3103 / \mathrm{S} 1060992 \mathrm{X} 10010042$.

101. Kazanskiy N, Skidanov R. Binary beam splitter. Applied Optics, 2012; 51(14): 2672-2677. DOI: 10.1364/AO.51.002672.

102. Kazanskiy NL, Popov SB. The distributed vision system of the registration of the railway train. Computer Optics, 2012; 36(3): 419-428.

103. Kazanskiy NL, Khonina SN, Skidanov RV, Morozov AA, Kharitonov SI, Volotovskiy SG. Formation of images using multilevel diffractive lens. Computer Optics, 2014; 38(3): 425 434.

104. Golub MA, Kazanskiy NL, Sissakian IN, Soifer VA, Kharitonov SI. Diffraction calculation for an optical element which focuses into a ring. Optoelectronics, Instrumentation and Data Processing, 1987; 23(6): 7-14.

105. Golub MA, Kazanskiy NL, Sissakian IN, Soifer VA, Kharitonov SI. Evaluation of the diffraction blur of the focal line of geometrical optics focusators. Computer Optics, 1989; 5: 34-38. [in Russian]

106. Khonina SN, Kazanskiy NL, Volotovsky SG. Influence of Vortex Transmission Phase Function on Intensity Distribution in the Focal Area of High-Aperture Focusing System. Optical Memory and Neural Networks (Information Optics), 2011; 20(1): 23-42. DOI: 10.3103/S1060992X11010024.

107. Golovashkin DL, Kasanskiy NL. Solving Diffractive Optics Problem using Graphics Processing Units. Optical Memory and Neural Networks (Information Optics), 2011; 20(2): 85-89. DOI: $10.3103 / \mathrm{S} 1060992 X 11020019$. 
108. Khonina SN, Kazanskii NL, Ustinov AV, Volotovskii SG. The lensacon: nonparaxial effects. Journal of Optical Technology, 2011; 78(11): 724-729. DOI: 10.1364/JOT.78.000724.

109. Kazanskiy NL, Kharitonov SI. Transmission of the space-limited broadband symmetrical radial pulses focused through a thin film. Computer Optics, 2012; 36(1): 4-13.

110. Khonina SN, Savelyev DA, Kazanskiy NL. Vortex phase elements as detectors of polarization state. Optics Express, 2015; 23(14): 17845-17859.

111. Kharitonov SI, Volotovsky SG, Khonina SN, Kazanskiy NL. Differential calculation method of diffraction of x-rays by crystal: scalar theory. Computer Optics, 2015; 39(4): 469-479.

112. Pozhar VE. Pustovoit VI, Kutuza IB, Perchik AV. Mazur MM, Shorin VN. Double AOTF Spectral Imaging System. Proceedings of SPIE, 2005; 5953: 200-203.

113. Kazanskiy NL, Kharitonov SI, Khonina SN, Volotovskiy SG, Strelkov YuS. Simulation of hyperspectrometer on spectral linear variable filters. Computer Optics, 2014; 2014; 38(2): 256-270.

114. Kazanskiy NL, Kharitonov SI, Karsakov AV, Khonina SN. Modeling action of a hyperspectrometer based on the Offner scheme within geometric optics. Computer Optics, 2014; 38(2): 271-280.

115. Kazanskiy NL, Kharitonov SI, Khonina SN. Simulation of a hyperspectrometer based on linear spectral filters using vector Bessel beams. Computer Optics, 2014; 38(4): 770-776.

116. Kazanskiy NL, Kharitonov SI, Doskolovich LL, Pavelyev AB. Modeling of the operation of hyperspectrometer based on the Offner scheme. Computer Optics, 2015; 39(1): 70-76. DOI: 10.18287/0134-2452-2015-39-1-70-76.

117. Greisukh GI, Danilov VA, Ezhov EG, Levin IA, Stepanov SA, Usievich BA. Comparison of electromagnetic and scalar methods for evaluation of efficiency of diffractive lenses for wide spectral bandwidth. Optics Communication, 2015; 338(1): 54-57.

118. Greisukh GI, Danilov VA, Ezhov EG, Stepanov SA, Usievich BA. Spectral and angular dependences of the efficiency of diffraction lenses with a dual-relief and two-layer microstructure. Journal of Optical Technology, 2015; 82(5): 308-311.

119. Greisukh GI, Danilov VA, Ezhov EG, Stepanov SA, Usievich BA. Spectral and angular dependences of the efficiency of relief-phase diffractive lenses with two- and three-layer microstructures. Optics and Spectroscopy, 2015; 118(6) 997-1004.

120. Petrov NI, Nikitin VG, Danilov VA, Popov VV, Usievich BA. Subwavelength diffractive color beam combiner. Applied Optics, 2014; 53(25): 5740-5744.

121. Krivoshlykov SG. Holographic recording of infrared diffractive optics based on ZnSe material. Applied Optics, 2015; 54(12): 3569-3575.

122. Soifer VA. Diffractive optics and nanophotonics. Computer Optics, 2008; 32(2): 110-118.

123. Kazanskiy NL, Serafimovich PG, Khonina SN. Harnessing the Guided-Mode Resonance to Design Nanooptical Transmission Spectral Filters. Optical Memory and Neural Networks (Information Optics), 2010; 19(4): 318-324. DOI: 10.3103/S1060992X10040090.

124. Bykov DA, Doskolovich LL, Soifer VA, Kazanskiy NL. Extraordinary Magneto-Optical Effect of a Change in the Phase of Diffraction Orders in Dielectric Diffraction Gratings. Journal of Experimental and Theoretical Physics, 2010; 111(6): 967-974. DOI: 10.1134/S1063776110120095.

125. Kazanskiy NL, Serafimovich PG, Khonina SN. Use of photonic crystal cavities for temporal differentiation of optical signals. Optics Letters, 2013; 38(7): 1149-1151. DOI: 10.1364/OL.38.001149. 
126. Kazanskiy NL, Serafimovich PG. Coupled-resonator optical wave-guides for temporal integration of optical signals. Optics Express, 2014; 22(11): 14004-14013. DOI: 10.1364/OE.22.014004.

127. Bykov DA, Doskolovich LL, Bezus EA, Soifer VA. Optical computation of the Laplace operator using phase-shifted Bragg grating. Optics Express, 2014; 22(21): 25084-25092.

128. Golovastikov NV, Bykov DA, Doskolovich LL. Spatial integration of optical beams using multilayer Bragg structures. Computer Optics, 2014; 38(3): 372-376.

129. Egorov AV, Kazanskiy NL, Serafimovich PG. Using coupled photonic crystal cavities for increasing of sensor sensitivity. Computer Optics, 2015; 39(2): 158-162. DOI: 10.18287/0134-2452-2015-39-2-158-162.

130. Serafimovich PG, Kazanskiy NL. Optical modulator based on coupled photonic crystal cavities // Journal of Modern Optics, 2016; 63(13): 1233-1238. DOI: 10.1080/09500340.2015.1135258.

131. Soifer VA. Diffractive nanophotonics and advanced information technologies. Bulletin of the Russian Academy of Sciences, 2014; 84(1): 11-24.

132. Kaminskaya TP, Popov VV, Saletsky AM. Characterization of the surface relief of film diffractive optical elements. Computer Optics, 2016; 40(2): 215-224. DOI: 10.18287/24126179-2016-40-2-215-224.

133. Firsov An, Firsov A, Loechel B, Erko A, Svintsov A, Zaitsev S. Fabrication of digital rainbow holograms and 3-D imaging using SEM based e-beam lithography. Optics Express, 2014; 22(23): 28756-28770.

134. Ageshin SF, Goncharsky AV, Popov VV. Optical filters for professional and amateur photography. Fotography, 1993; 2: 34-36. 